\title{
Multivariable Three-Term Optimal Controller Design for Large-Scale Systems
}

\author{
Edward J. Davison, Daniel E. Davison, Simon Lam
}

\begin{abstract}
This paper deals with the design of controllers for large-scale linear time-invariant multi-input multi-output systems, such as those that often arise in process control. We focus on two standard controller objectives: (i) asymptotic regulation subject to unmeasurable constant disturbances, and/or (ii) asymptotic tracking of constant set-points. In either case, standard output-feedback controller design methodologies typically result in controllers that have order at least as large as that of the plant; for large-scale systems, the controller order can consequently be impractically large. The purpose of this paper is to introduce, for the subclass of plants that are open-loop stable, a low-order three-term (i.e., PID) multivariable control design approach that is practical to compute numerically, even for large-scale systems. A design algorithm and existence results to construct such a controller are given, and the approach is applied to several examples. Remarkably, at least for the examples considered, the three-term controller's performance is quite similar to that achieved by the standard (much higher order) controller that solves the servomechanism problem.
\end{abstract}

\section{INTRODUCTION}

Engineers have long known the benefits of integral feedback control for tracking constant reference signals in the presence of unknown constant disturbances. Indeed, proportional-integral (PI) and proportional-integral-derivative (PID) controllers are among the most popular controllers found in industrial process control. There is a rich literature on the design and tuning of PID controllers for singleinput single-output (SISO) systems (e.g., see [1]-[5] and the references therein).

For multiple-input multiple-output (MIMO) systems, the design of multivariable controllers with integral action can be found in a number of early papers, including some written by the first author (e.g., see [6]-[10]). The existing techniques usually either (i) assume that full-state information is available, or (ii) require an observer to estimate the state. In the case where the plant order is large, the first situation results in an expensive and impractical sensing burden. The second situation is just as undesirable if the plant order is large since the order of the resulting controller is at least as large as that of the plant. Hence, for large industrial systems, many MIMO controller design approaches are impractical. It is therefore understandable that both academic and industrial researchers have sought for, and continue to search for, MIMO design procedures that result in low-order controllers.

This work has been supported by NSERC under grant No. A4396.

E. J. Davison and S. Lam are with the Systems Control Group, Department of Electrical and Computer Engineering, University of Toronto, Ontario, Canada, M5S 3G4 \{simon, ted\} @ control. utoronto. ca

D. E. Davison is with the Department of Electrical and Computer Engineering, University of Waterloo, Ontario, Canada, N2L 3G1 ddavisoneuwaterloo.ca
Inspiring by their success in classical SISO problems, the most popular MIMO low-order controllers are PID controllers. Various tuning methods have been developed for MIMO PID control (e.g., see [1], [11] and the references therein). However, many of these design procedures require that the MIMO plant has special properties. For instance, some papers assume that the system is decoupled or can be decoupled, while others assume that the system can be approximated by a low-order system with delay. Fortunately, by recognizing that there exist systems where the inter-loop interactions are not necessarily small and where the system cannot be approximated by a low-order system with delay, various researchers have managed to devise MIMO PID design procedures without making restrictive assumptions on the structure of the system. For example, in [12], a technique using pole placement is proposed which allows the designer to partially place some of the poles of the closedloop system; if the remaining poles are unstable or unsatisfactory, then the design parameters are modified and the pole-placement problem is solved again. Another approach is taken in [13], where a MIMO optimal PID controller is obtained by numerically solving several simultaneous nonlinear matrix equations. In [14], a third approach for the design of an optimal PID controller is proposed, which involves solving a high-order static state-feedback problem and then reducing the size of the controller by retaining the "dominant" dynamics of the closed-loop system. Finally, in [15] and [16], a MIMO PID controller is obtained by solving a similar static output-feedback problem using linear matrix inequality (LMI) methods. A disadvantage with all of these techniques is that the controller synthesis requires solving various subproblems multiple times before obtaining a stabilizing controller (if one exists), and therefore the complexity of the procedures increases greatly with the system size. Moreover, in almost all approaches, a stabilizing solution is not even guaranteed to exist.

In this paper, we describe a new optimal PID controller design approach for MIMO open-loop stable plants. The approach is based on the multivariable tuning regulator of [17], using the parameter optimization methods of [18] and [19], and contains only three scalar parameters to be optimized based on a quadratic performance criterion. Several practical advantages of the proposed technique stand out: (i) the approach is suitable for large-scale systems because the optimization dimension of the optimization parameter space, $\mathbb{R}^{3}$, is relatively small and independent of the system size; (ii) we have been able to characterize the conditions under which a solution to the optimization problem exist (namely, 
a solution exists if and only if a solution to the underlying servomechanism problem exists); and (iii) a starting point to the parameter optimization problem can always be found to guarantee a solution to the problem.

This paper is organized as follows. Section II describes the structure and existence conditions of the proposed multivariable three-term controller, and the procedure for designing such a controller. Then Section III investigates the performance of the proposed three-term controller by applying the technique to several different systems and comparing the closed-loop performance to that which is achieved by designing full-order optimal servo controllers.

\section{THREE-TERM OPTIMAL CONTROLLER DESIGN}

Consider the open-loop asymptotically stable linear timeinvariant (LTI) system, which may or may not be minimum phase:

$$
\begin{aligned}
\dot{x} & =A x+B u+E w \\
y & =C x+F w \\
e & =y_{\text {ref }}-y,
\end{aligned}
$$

where $x \in \mathbb{R}^{n}$ is the state of the system, $u \in \mathbb{R}^{m}$ is the input, $y \in \mathbb{R}^{r}$ is the output, $w \in \mathbb{R}^{\Omega}$ is an unmeasurable constant disturbance, $e \in \mathbb{R}^{r}$ is the error, and $y_{\text {ref }} \in \mathbb{R}^{r}$ is a constant tracking signal. The control objective is to solve the robust servomechanism problem (RSP) [20] for this system.

Start by introducing the notation $(\cdot)^{+}=(\cdot)^{T}\left[(\cdot)(\cdot)^{T}\right]^{-1}$ and recalling the following two fundamental results, from [17], concerning the solution to the RSP:

Lemma 2.1: [17] There exists a solution to the RSP for (1) if and only if $\operatorname{rank}\left(C A^{-1} B\right)=r$.

Lemma 2.2: [17] Consider the MIMO integral controller $\dot{\eta}=e, u=-\epsilon\left(C A^{-1} B\right)^{+} \eta$ (where $\epsilon>0$ is a design parameter) applied to the plant (1). Then $\operatorname{rank}\left(C A^{-1} B\right)=r$ is a necessary and sufficient condition for the existence of an $\epsilon^{*}>0$ such that the closed-loop system is asymptotically stable for all $\epsilon \in\left(0, \epsilon^{*}\right)$.

Given these two results, throughout the paper it is assumed that $\operatorname{rank}\left(C A^{-1} B\right)=r$, which implies $m \geq r$.

\section{A. Controller structure}

To control (1), we choose a multivariable three-term controller structure of the form

$$
u=\epsilon_{I} \mathcal{S} \eta+\epsilon_{P} \mathcal{S} e+\epsilon_{D} \mathcal{S} \dot{e}
$$

where

$$
\dot{\eta}=e, \quad \mathcal{S}:=-\left(C A^{-1} B\right)^{+}
$$

and where $\epsilon_{I}, \epsilon_{P}$, and $\epsilon_{D}$ are scalar tuning parameters of the controller. The three-term controller (2) can be considered to be a generalization of the "tuning controller" introduced in [17] and described in Lemma 2.2. Lemma 2.2 can be readily extended to show that sufficiently small choices for the three parameters in controller (2) guarantees closed-loop stability:

Lemma 2.3: Let the controller (2) be applied to (1); then there exist $\epsilon^{*}>0$ and $\epsilon^{* *}>0$ such that the closed-loop system is asymptotically stable for all $\epsilon_{I} \in\left(0, \epsilon^{*}\right), \epsilon_{P} \in$ $\left(0, \epsilon^{* *}\right)$, and $\epsilon_{D} \in\left(0, \epsilon^{* *}\right)$.

\section{B. Optimal parameter selection}

We propose that the three parameters $\epsilon_{I}, \epsilon_{P}$, and $\epsilon_{D}$ be chosen by solving an optimization problem. In practice, problems such as the one described below can be solved numerically using the approach of [18] and [19].

Motivated by the "optimal transient shaping" results in [21], we introduce the performance index

$$
J_{\epsilon_{I}, \epsilon_{P}, \epsilon_{D}}=\int_{0}^{\infty}\left(z^{T} z+\epsilon \dot{u}^{T} \dot{u}\right) d \tau
$$

where $z:=\theta \dot{e}+e, \theta>0$, and $\epsilon>0$. As discussed in [21], the above performance index is appealing because making $J_{\epsilon_{I}, \epsilon_{P}, \epsilon_{D}}$ small results in "small" $z$, which implies $e$ decays roughly as a first-order system with settling time determined by $\theta$. For the problem at hand, the goal is to minimize (with respect to the three parameters $\epsilon_{I}, \epsilon_{P}$, and $\epsilon_{D}$ ) performance index (4) subject to the constraint

$$
\begin{aligned}
{\left[\begin{array}{c}
\ddot{x} \\
\dot{e}
\end{array}\right] } & =\left[\begin{array}{cc}
A & 0 \\
-C & 0
\end{array}\right]\left[\begin{array}{c}
\dot{x} \\
e
\end{array}\right]+\left[\begin{array}{c}
B \\
0
\end{array}\right] \dot{u} \\
z & =\left[\begin{array}{ll}
-\theta C & I
\end{array}\right]\left[\begin{array}{c}
\dot{x} \\
e
\end{array}\right] .
\end{aligned}
$$

Substitute the controller equation (2) into (5) to obtain, for $\tilde{x}:=\left[\begin{array}{c}\dot{x} \\ e\end{array}\right]$, the closed-loop equations

$$
\begin{aligned}
\dot{\tilde{x}} & =\tilde{\mathcal{A}} \tilde{x} \\
z & =\tilde{C} \tilde{x} \\
\dot{u} & =\tilde{C}_{u} \tilde{x}
\end{aligned}
$$

where, on defining $\mathcal{T}:=\left(I+\epsilon_{D} \mathcal{S} C B\right)^{-1} \mathcal{S}$,

$$
\begin{aligned}
\tilde{\mathcal{A}} & =\left[\begin{array}{cc}
A-B \mathcal{T}\left(\epsilon_{P} C+\epsilon_{D} C A\right) & \epsilon_{I} B \mathcal{T} \\
-C & 0
\end{array}\right] \\
\tilde{C} & =\left[\begin{array}{cc}
-\theta C & I
\end{array}\right] \\
\tilde{C}_{u} & =\left[\begin{array}{ll}
-\mathcal{T}\left(\epsilon_{P} C+\epsilon_{D} C A\right) & \epsilon_{I} \mathcal{T}
\end{array}\right] .
\end{aligned}
$$

Note that the gain matrix $\mathcal{T}$ is well defined for almost all $\epsilon_{D}$; we disallow values of $\epsilon_{D}$ where $\operatorname{det}\left(I+\epsilon_{D} \mathcal{S} C B\right)=0$. Next, substitute for $z$ and $\dot{u}$ from (6) into (4), yielding (dropping the $\epsilon_{I}, \epsilon_{P}, \epsilon_{D}$ subscript for convenience)

$J=\tilde{x}^{T}(0)\left[\int_{0}^{\infty} e^{\tilde{\mathcal{A}}^{T} \tau}\left(\tilde{C}^{T} \tilde{C}+\epsilon \tilde{C}_{u}^{T} \tilde{C}_{u}\right) e^{\tilde{\mathcal{A}} \tau} d \tau\right] \tilde{x}(0)$

or

$$
J=\tilde{x}^{T}(0) \Gamma \tilde{x}(0)
$$

where $\Gamma>0$ is the solution of the Lyapunov equation

$$
\tilde{\mathcal{A}}^{T} \Gamma+\Gamma \tilde{\mathcal{A}}=-\left(\tilde{C}^{T} \tilde{C}+\epsilon \tilde{C}_{u}^{T} \tilde{C}_{u}\right) .
$$

Assume the controller is initialized so that $u(0)=0$. Hence, $\tilde{x}(0)=\left[\begin{array}{ccc}A & E & 0 \\ -C & -F & I\end{array}\right]\left[\begin{array}{c}x(0) \\ w \\ y_{r e f}\end{array}\right]$. The expression in (9) can then be simplified in three cases of most interest:

1. Disturbance rejection $\left(x(0)=0, w \neq 0, y_{\text {ref }}=0\right)$ :

$$
J_{w}:=J=\operatorname{trace}\left(\left[\begin{array}{ll}
E^{T} & -F^{T}
\end{array}\right] \Gamma\left[\begin{array}{c}
E \\
-F
\end{array}\right]\right)
$$



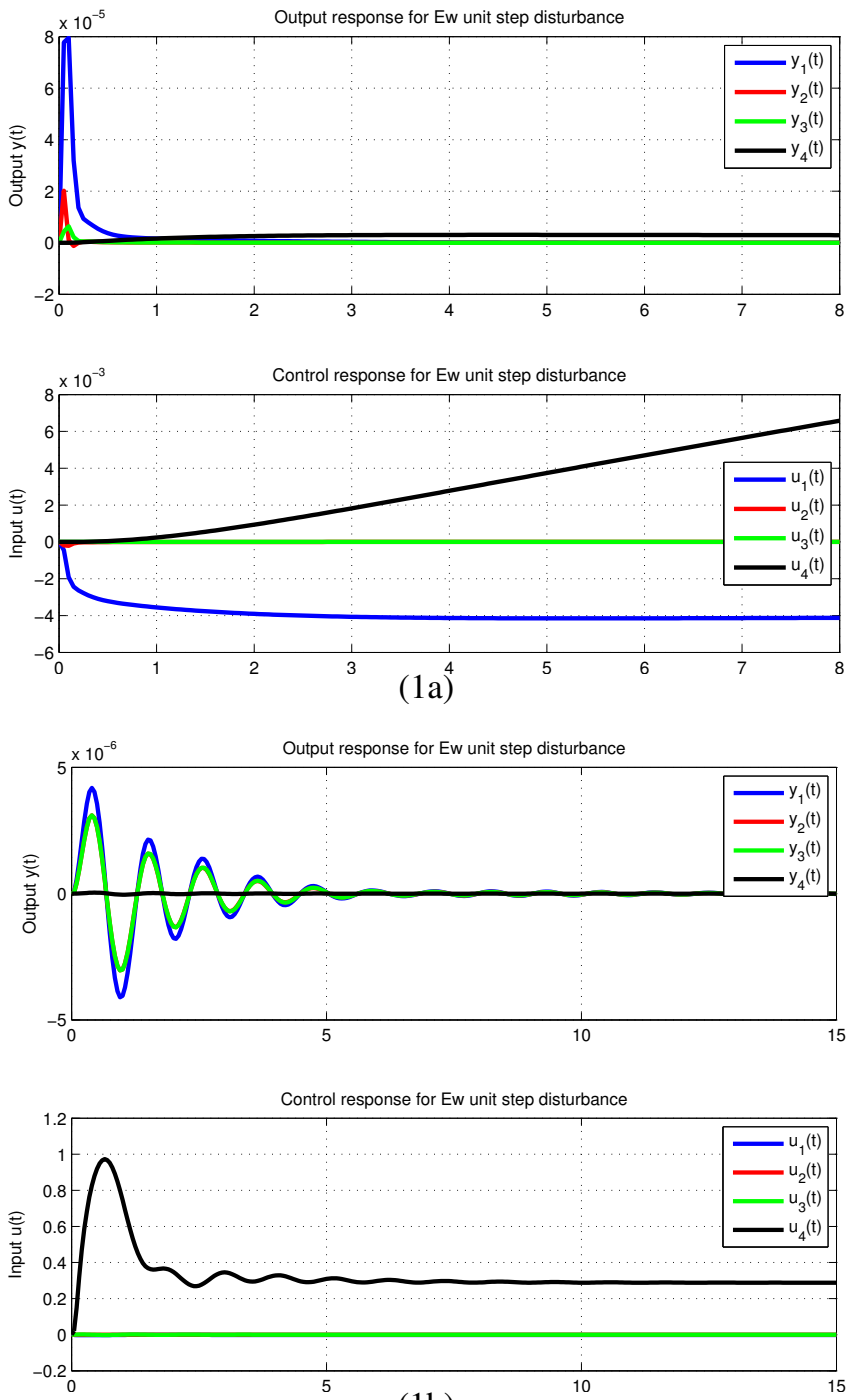

(1b)

Fig. 1. Plant output and control signal of the chemical plant versus time (in seconds) with $w=[1,0,0]^{T}$ using (a) the $45^{t h}$ order servomechanism controller, and (b) the proposed $4^{\text {th }}$ order PID controller, both with $\epsilon=$ $10^{-5}$ and $\theta=0$. Note that the $u_{4}(t)$ signal in part (a) of the figure settles to $\approx 0.3$ at time $\approx 1800 \mathrm{~s}$.

Using this performance index measures the "average cost" of (4) over all disturbances $w$ uniformly distributed on an unit disk ball [18]. Likewise we choose:

2. Tracking $\left(x(0)=0, w=0, y_{\text {ref }} \neq 0\right)$ :

$$
J_{y_{r e f}}:=J=\operatorname{trace}\left(\left[\begin{array}{ll}
0 & I
\end{array}\right] \Gamma\left[\begin{array}{l}
0 \\
I
\end{array}\right]\right)
$$

3. Regulation $\left(x(0) \neq 0, w=0\right.$, $\left.y_{\text {ref }}=0\right)$ :

$$
J_{x_{0}}:=J=\operatorname{trace}\left(\left[\begin{array}{ll}
A^{T} & -C^{T}
\end{array}\right] \Gamma\left[\begin{array}{c}
A \\
-C
\end{array}\right]\right)
$$

In summary, to determine the optimal values of the controller parameters $\epsilon_{I}, \epsilon_{P}$, and $\epsilon_{D}$, the following nonlinear parameter optimization problem is to be solved:

$$
\begin{array}{ll}
\min _{\epsilon_{I}, \epsilon_{P}, \epsilon_{D}} J_{\epsilon_{I}, \epsilon_{P}, \epsilon_{D}} & \begin{array}{l}
\text { subject to the constraint } \\
\text { that the closed-loop }
\end{array} \\
& \text { system (6) is stable }
\end{array}
$$
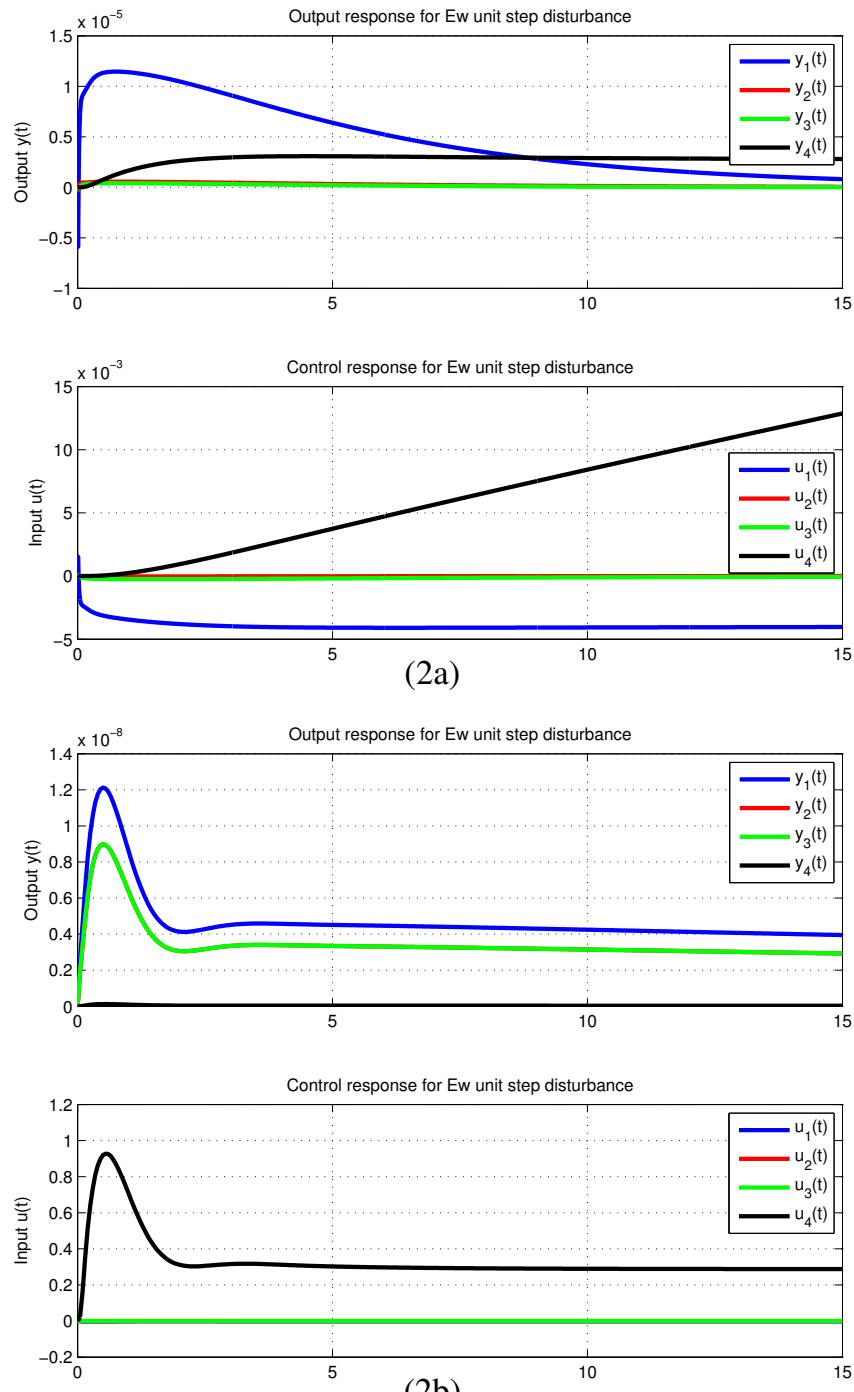

(2b)

Fig. 2. The same as Figure 1 except with $\theta=5$ instead of $\theta=0$. Note that the transient response is now significantly smoother.

where, depending on the problem definition, $J_{\epsilon_{I}, \epsilon_{P}, \epsilon_{D}}$ is chosen to be $J_{w}, J_{y_{\text {ref }}}$, or $J_{x_{0}}$ (or any linear combination of these indices). In the examples studied in Section III, the choice of $J_{w}$ was used in (14) since disturbance rejection is the most important problem in process control.

Remark 2.1: It may well be that multiple local minima exist for the optimization problem (14). From an engineering viewpoint, all things being equal, the solution of the optimal parameter vector $\left(\epsilon_{I}, \epsilon_{P}, \epsilon_{D}\right)$ that has the smallest norm is desirable, which implies that a starting point "as close as possible" to the origin should be used.

\section{Discussion of optimization starting point}

It follows from Lemma 2.3 that there exists a feasible starting point for the optimization problem (14) given by $\epsilon_{I}$, $\epsilon_{P}=0$, and $\epsilon_{D}=0$ with $\epsilon_{I}>0$ "sufficiently small". For the examples in Section III, the choice of performance index $J_{w}$ with $\left(\epsilon_{I}, \epsilon_{P}, \epsilon_{D}\right)=\left(10^{-5}, 10^{-8}, 10^{-12}\right)$ was used as the starting point. 


\section{NUMERICAL EXAMPLES}

In this section we compare the disturbance rejection performance of the PID controller proposed in Section II with that of a general servomechanism controller [20]. We also briefly compare the robustness of the two control schemes using the real stability radius, denoted $r_{\text {stab }}$ [22]. (See the Appendix for the definition of $r_{s t a b}$.) Three systems are considered below: the Williams and Otto chemical plant in [23], the distillation column with pressure variation in [24], and a $4^{\text {th }}$ order example in [12].

\section{A. Williams and Otto chemical plant [23]}

The Williams and Otto chemical plant in [23] is a $41^{\text {st }}$ order chemical plant model with 4 inputs, 4 outputs, and 3 disturbance channels. The system is open-loop stable and minimum-phase. Following the procedure in Section II, the inverse of the open-loop input steady-state gain matrix (3) was found to be:

$$
\mathcal{S}=\left[\begin{array}{cccc}
10.66 & 0 & 0 & -800.1 \\
-2.860 & 3.860 & 0 & 0 \\
0 & -1.642_{10^{3}} & 1.642_{10^{3}} & 0 \\
1.059_{10^{-9}} & -1.098_{10^{-9}} & 0 & -9.960_{10^{4}}
\end{array}\right]
$$

Then using the starting point $\left(\epsilon_{I}^{0}, \epsilon_{P}^{0}, \epsilon_{D}^{0}\right)=$ $\left(10^{-5}, 10^{-8}, 10^{-12}\right)$, the optimization problem (14) was solved with $J_{\epsilon_{I}, \epsilon_{P}, \epsilon_{D}}=J_{w}, \theta=0$, and $\epsilon=10^{-5}$, yielding an optimal controller with structure (2) and parameter values

$$
\left(\epsilon_{I}, \epsilon_{P}, \epsilon_{D}\right)=(985.1,16.99,25.88)
$$

and

$$
\mathcal{T}=\left[\begin{array}{cccc}
1.449_{10^{-2}} & 0 & 0 & -0.1241 \\
-3.888_{10^{-3}} & 3.860 & 0 & -24.47 \\
0 & -4.862 & 4.862 & 0 \\
0 & 0 & 0 & -1.137_{10^{4}}
\end{array}\right]
$$

As summarized in Table I, the controller obtained is $4^{\text {th }}$ order, and achieves an optimal disturbance cost, $J_{w}$, of $6.520_{10^{-5}}$ with a real stability radius, $r_{s t a b}$, of $1.312_{10^{-6}}$. For comparison, a $45^{\text {th }}$-order servomechanism controller for the same plant was obtained by minimizing (4) with $\epsilon=10^{-5}$ and $\theta=0$, and it achieves a cost of $1.092_{10^{-8}}$ with a stability radius of $1.004_{10^{-5}}$. Both types of controllers were then redesigned using $\theta=5$ (holding $\epsilon=10^{-5}$ ). In this case, the new three-term optimal controller parameter values are

$$
\left(\epsilon_{I}, \epsilon_{P}, \epsilon_{D}\right)=(983.3,473.3,1.691) .
$$

Figures 1 and 2 illustrate the behavior of the four controllers for the unit step disturbance $w=[1,0,0]^{T}$ with $\theta=0$ and $\theta=5$, respectively. It can be seen from these figures that, to our surprise, the proposed three-term controller performs reasonably well in comparison to the $45^{\text {th }}$-order servomechanism controller; we had expected that, due to the massive decrease in controller order, performance would suffer greatly. In addition, as we would expect because of the chosen structure of the performance index (4), the controller with $\theta=5$ produces a more desirable transient response,
TABLE I

SUMMARY OF CONTROLLERS FOR WILLIAMS AND OTTO CHEMICAL PLANT [23] WITH $\theta=0$

\begin{tabular}{l|c|c}
\hline \hline & Servocontroller & PID controller \\
\hline Controller order & 45 & 4 \\
Cost $J_{w}$ & $1.092_{10^{-8}}$ & $6.520_{10^{-5}}$ \\
$r_{\text {stab }}$ & $1.004_{10^{-5}}$ & $1.312_{10^{-6}}$ \\
\hline \hline
\end{tabular}

TABLE II

SUMMARY OF CONTROLLERS FOR DISTILLATION COLUMN [24] WITH $\theta=0$

\begin{tabular}{l|c|c}
\hline \hline & Servocontroller & PID controller \\
\hline Controller order & 14 & 3 \\
Cost $J_{w}$ & $2.994_{10^{-7}}$ & $7.064_{10^{-4}}$ \\
$r_{\text {stab }}$ & $1.604_{10^{-4}}$ & $2.654_{10^{-4}}$ \\
\hline \hline
\end{tabular}

with no oscillations and greater disturbance rejection compared to the case when $\theta=0$.

\section{B. Distillation column with pressure variation [24]}

The distillation column studied in [24] is an $11^{\text {th }}$-order industrial plant that is open-loop stable and minimum-phase, with a single disturbance corresponding to the input feed composition. Matrix $\mathcal{S}$ in (3) was found to be:

$$
\mathcal{S}=\left[\begin{array}{ccc}
-156.9 & 184.0 & 24.99 \\
101.5 & -239.5 & 15.23 \\
-5.012_{10^{-2}} & 0.3019 & 3.327_{10^{-3}}
\end{array}\right]
$$

Starting with $\left(\epsilon_{I}^{0}, \epsilon_{P}^{0}, \epsilon_{D}^{0}\right)=\left(10^{-5}, 10^{-8}, 10^{-12}\right)$, the following optimal controller was found by solving (14), with $J_{\epsilon_{I}, \epsilon_{P}, \epsilon_{D}}=J_{w}, \theta=0$, and $\epsilon=10^{-5}$ in (4):

$$
\mathcal{T}=\left[\begin{array}{ccc}
-1.581_{10^{2}} & 1.839_{10^{2}} & 25.53 \\
1.014_{10^{2}} & -2.402_{10^{2}} & 15.46 \\
-5.037_{10^{-2}} & 0.3020 & 3.408_{10^{-3}}
\end{array}\right]
$$

and

$$
\left(\epsilon_{I}, \epsilon_{P}, \epsilon_{D}\right)=\left(4.902,2.995_{10^{3}}, 0.9925\right) .
$$

The controller is $3^{\text {rd }}$ order, with an optimal disturbance cost $J_{w}$, of $7.064_{10^{-4}}$. In comparison, a standard servomechanism controller for the same plant is $14^{\text {th }}$ order, and achieves a performance index $J_{w}$ of $2.994_{10^{-7}}$. A comparsion of the two controllers is made in Table II and, for response to a unit step disturbance, in Figure 3. As with the chemical plant example, despite the huge reduction in controller order, the proposed PID controller achieves a response and robustness that are comparable to that of the servo controller.

\section{Example from [12]}

The following unstable system with an unstable pole located at $s=1$, is taken from [12]:

$$
\begin{aligned}
& A=\left[\begin{array}{cccc}
0 & 1 & 0 & 0 \\
0 & 0 & 1 & 0 \\
0 & 0 & 0 & 1 \\
10 & 2 & -9 & -2
\end{array}\right] B=\left[\begin{array}{ll}
0 & 1 \\
0 & 0 \\
0 & 2 \\
1 & 0
\end{array}\right] E=\left[\begin{array}{ll}
1 \\
-1 \\
0 \\
0
\end{array}\right] \\
& C=\left[\begin{array}{llll}
1 & 0 & 0 & 0 \\
0 & 1 & 0 & 0
\end{array}\right] F=\left[\begin{array}{l}
0 \\
0
\end{array}\right] .
\end{aligned}
$$



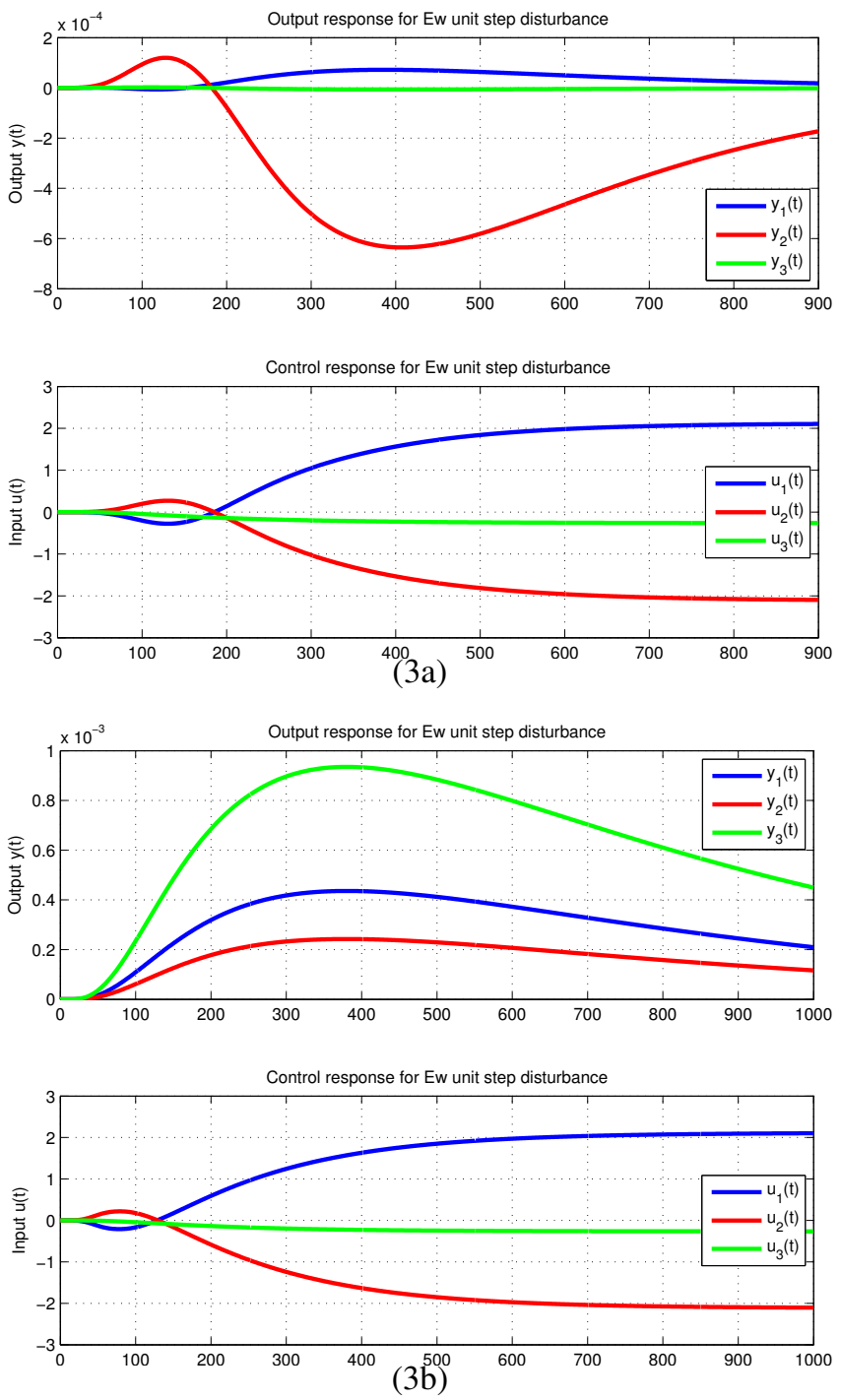

Fig. 3. Plant output and control signal of the distillation column versus time (in seconds) with $w=1$ using (a) the $14^{\text {th }}$ order servomechanism controller with $\theta=0$, and (b) the proposed $3^{\text {rd }}$ order PID controller with $\theta=0$.

The plant must first be stabilized for the results of this paper to be applicable. On noting that the system can be stabilized using the proportional controller $u_{2}=-5 y_{2}$, the following optimal three-term controller is obtained using $\epsilon=10^{-5}$ and $\theta=0$ :

$$
T=\left[\begin{array}{cc}
-10 & 57.0 \\
0 & 4
\end{array}\right]
$$

and

$$
\left(\epsilon_{I}, \epsilon_{P}, \epsilon_{D}\right)=(14.77,3.679,1.375)
$$

Table III and Figure 4 compares the performance of the PID controller obtained in [12] with that proposed in this paper. It can be seen that the three-term controller works quite well even in the presence of an unstable open-loop pole.

\section{Addressing a potential criticism}

One might conjecture that the three-term controller's performance is similar to that of the full-order servo controller only because both control schemes yield poor performance compared to the "no control" (i.e., open loop) performance; in other words, it is conceivable that the full-order servo controller's performance is poor, and therefore the comparisons we make for the chemical plant system and the distillation column system are not of interest. In fact, all of the controllers designed above are highly effective compared to the open-loop performance. As evidence, Table IV lists the output steady-state errors of the two industrial systems when no control is applied and the systems are subject to constant unit disturbances. Comparing this data with the peak output values apparent from Figures 1-2 indicates clearly the effectiveness of the feedback control schemes.

\section{Conclusions}

An optimal PID controller is proposed for controlling large-scale open-loop stable multivariable plants. Like all multivariable PID controllers, the control order is low, which is an advantage compared to the high-order controllers that many other conventional design techniques generate. A key strength of this work is that the proposed three-term controller is easily tuned, even for large-scale systems with many inputs and outputs, since the controller contains only three scalar parameters to be optimized. The design approach in this paper was illustrated by synthesizing $4^{\text {th }}$ and $3^{\text {rd }}$ order three-term controllers for two industrial examples; the disturbance rejection performance was found to be comparable to that achieved by full-order servo controllers, which are of $45^{\text {th }}$ and $14^{\text {th }}$ order, respectively. It was also shown how the transient response of the closed-loop system can be improved by selecting $\theta>0$ in the performance index (4). Lastly, it can be seen from the third example studied in the paper that the assumption about the plant being open-loop stable is not essential.

\section{REFERENCES}

[1] K. K. Tan, Q. G. Wang, and C. C. Hang, Advances in PID Control. London: Springer, 1999.

[2] A. Datta, M.-T. Ho, and S. P. Bhattacharyya, Structure and Synthesis of PID Controllers. New York: Springer, 2000.

[3] M. T. Söylemez, N. Munro, and H. Baki, "Fast calculation of stabilizing PID controllers," Automatica, vol. 39, no. 1, pp. 121-126, 2003.

[4] "Special issue on PID," in IEEE Control Syst. Mag., vol. 26, no. 1, February 2006.

[5] L. H. Keel and S. P. Bhattacharyya, "Controller synthesis free of analytical models: Three terms controllers," IEEE Trans. Automat. Contr., vol. 53, no. 6, pp. 1353-1369, July 2008.

[6] E. J. Davison and H. W. Smith, "Pole assignment in linear time invariant multivariable systems with constant disturbances," Automatica, vol. 7, no. 4, pp. 489-498, July 1971.

[7] H. W. Smith and E. J. Davison, "The design of industrial regulators: integral feedback and feedforward control," Proc IEE, vol. 119, no. 8, pp. 1210-1216, August 1972.

[8] E. J. Davison, "The feedforward control of linear multivariable timeinvariant systems," Automatica, vol. 9, no. 5, pp. 561-573, September 1973.

[9] —_, "The feedforward and feedback control of a general servomechanism problem, part I," in 11th Annual Allerton Conference on Circuits \& System Theory, October 1973, pp. 343-352.

[10] _ - "The feedforward and feedback control of a general servomechanism problem, part II," in 11th Annual Allerton Conference on Circuits \& System Theory, October 1973, pp. 353-362. 
[11] Q. G. Wang, B. Zou, T. H. Lee, and Q. Bi, "Auto-tuning of multivariable PID controllers from decentralized relay feedback," Automatica, vol. 33, no. 3, pp. 319-330, 1997.

[12] H. Seraji and M. Tarokh, "Design of PID controllers for multivariable systems," Int. J. of Control, vol. 26, no. 1, pp. 75-83, 1977.

[13] S. Fukata, A. Mohri, and M. Takata, "On the determination of the optimal feedback gains for multivariable linear systems incorporating integral action," Int. J. of Control, vol. 31, no. 6, pp. 1027-1040, June 1980.

[14] J. Medanić and Z. Uskoković, "The design of optimal output regulators for linear multivariable systems with constant disturbances," Int. J. of Control, vol. 37, no. 4, pp. 809-830, 1983.

[15] F. Zheng, Q. G. Wang, and T. H. Lee, "On the design of multivariable PID controllers via LMI approach," Automatica, vol. 28, no. 3, pp. 517-526, 2002.

[16] C. Lin, Q. G. Wang, and T. H. Lee, "An improvement on multivariable PID controller design via iterative LMI approach," Automatica, vol. 40, no. 3, pp. 519-525, 2004.

[17] E. J. Davison, "Multivariable tuning regulators: the feedforward and robust control of a general servomechanism problem," IEEE Trans. Automat. Contr., vol. AC-21, no. 1, pp. 35-47, February 1976.

[18] E. J. Davison and I. Ferguson, "The design of controllers for the multivariable robust servomechanism problems using parameter optimization methods," IEEE Trans. Automat. Contr., vol. AC-26, no. 1, pp. 93-110, February 1981.

[19] E. J. Davison and T. N. Chang, "Decentralized controller design using parameter optimization methods," Control: Theory and Advanced Technology, Special Issue on Large Scale and Complex Systems, vol. 2, no. 2, pp. 131-154, June 1986, (invited paper).

[20] E. J. Davison and A. Goldenberg, "The robust control of a general servomechanism problem: the servo compensator," Automatica, vol. 11, pp. 461-471, 1975.

[21] D. E. Davison and E. J. Davison, "Optimal transient response shaping of the servomechanism problem," J. Optimization Theory \& Applications (JOTA), vol. 115, no. 3, pp. 491-515, December 2002.

[22] L. Qiu, B. Bernhardsson, A. Rantzer, E. J. Davison, P. M. Young, and J. C. Doyle, "A formula for computation of the real stability radius," Automatica, vol. 31, no. 6, pp. 879-890, 1996.

[23] T. J. Williams and R. E. Otto, "A generalized chemical model for the investigation of computer control," AIEE Trans. Part I (Comm. \& Electronics), vol. 79, pp. 458-473, November 1960.

[24] E. J. Davison, "Control of a distillation column with pressure variation," Trans. Instn. Chem. Engineering, vol. 45, no. 6, pp. 228-250, 1967.

\section{APPENDIX: $r_{s t a b}$ AS A MEASURE OF ROBUSTNESS}

Consider a system $\dot{x}=A x+B u, y=C x$ that is subject to the following singular perturbation (where $\bar{A}$ is assumed to be stable):

$$
\begin{aligned}
\left(\begin{array}{c}
\dot{x} \\
\epsilon \dot{\eta}
\end{array}\right) & =\left[\begin{array}{cc}
A & 0 \\
0 & \bar{A}
\end{array}\right]\left(\begin{array}{l}
x \\
\eta
\end{array}\right)+\left(\begin{array}{c}
B \\
\bar{B}
\end{array}\right) u \\
y & =\left[\begin{array}{ll}
C & \bar{C}
\end{array}\right]\left(\begin{array}{l}
x \\
\eta
\end{array}\right) .
\end{aligned}
$$

In the limit as $\epsilon \rightarrow 0$, the perturbed system simplifies to

$$
\begin{aligned}
\dot{x} & =A x+B u \\
y & =C x+\Delta_{D} u
\end{aligned}
$$

where $\Delta_{D}=-\bar{C} \bar{A}^{-1} \bar{B}$. We now consider $\Delta_{D}$ to be a completely uncertain matrix and define the real stability radius of $\Delta_{D}$, denoted $r_{s t a b}$, to be the largest bound such that the perturbed closed-loop system is stable for all $\left\|\Delta_{D}\right\|_{2}<$ $r_{s t a b}$. Note that $r_{s t a b}$ is a function of the controller. An algorithm to compute $r_{\text {stab }}$ is available in [22].
TABLE III

SUMMARY OF CONTROLLERS FOR EXAMPLE FROM [12]

\begin{tabular}{l|c|c}
\hline \hline & [12]'s controller & PID controller \\
\hline Controller order & 2 & 2 \\
Cost $J_{w}$ & - & 0.1687 \\
$r_{\text {stab }}$ & - & $9.036_{10^{-3}}$ \\
\hline \hline
\end{tabular}

TABLE IV

OPEN-LOOP STEADY-STATE OUTPUTS FOR VARIOUS INPUT DISTURBANCES

\begin{tabular}{l|cc}
\hline \hline Plant & Disturbance $w$ & Steady-state output $y(\infty)$ \\
\hline $\begin{array}{l}\text { Chemical } \\
\text { plant }\end{array}$ & $w=\left[\begin{array}{l}1 \\
0 \\
0\end{array}\right]$ & $y(\infty)=\left[\begin{array}{l}3.871_{10^{-4}} \\
2.869_{10^{-4}} \\
2.869_{10^{-4}} \\
2.889_{10^{-6}}\end{array}\right]$ \\
$\begin{array}{l}\text { Distillation } \\
\text { column }\end{array}$ & $w=1$ & $y(\infty)=[2.116,1.176,4.537]^{T}$ \\
\hline \hline
\end{tabular}
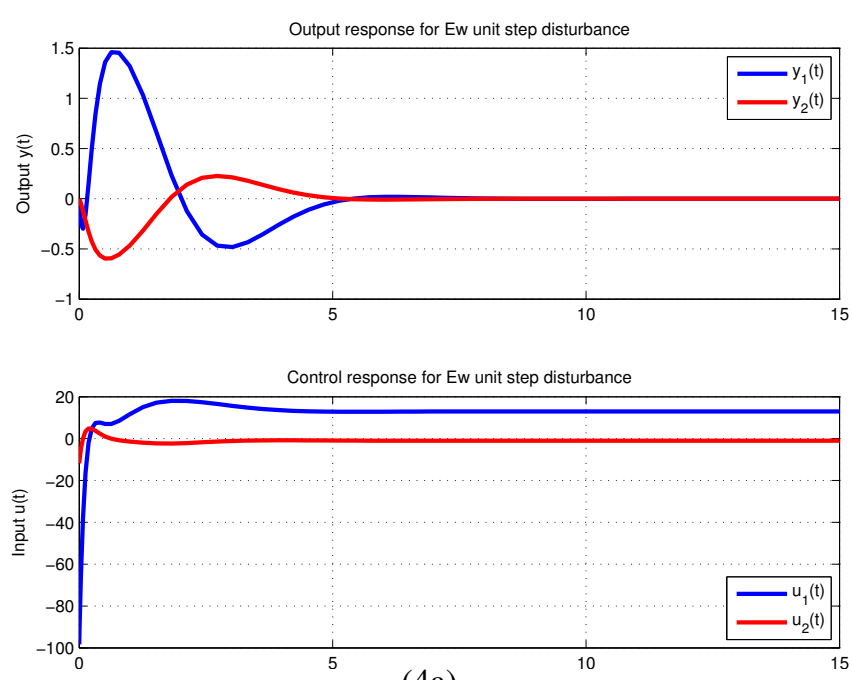

(4a)
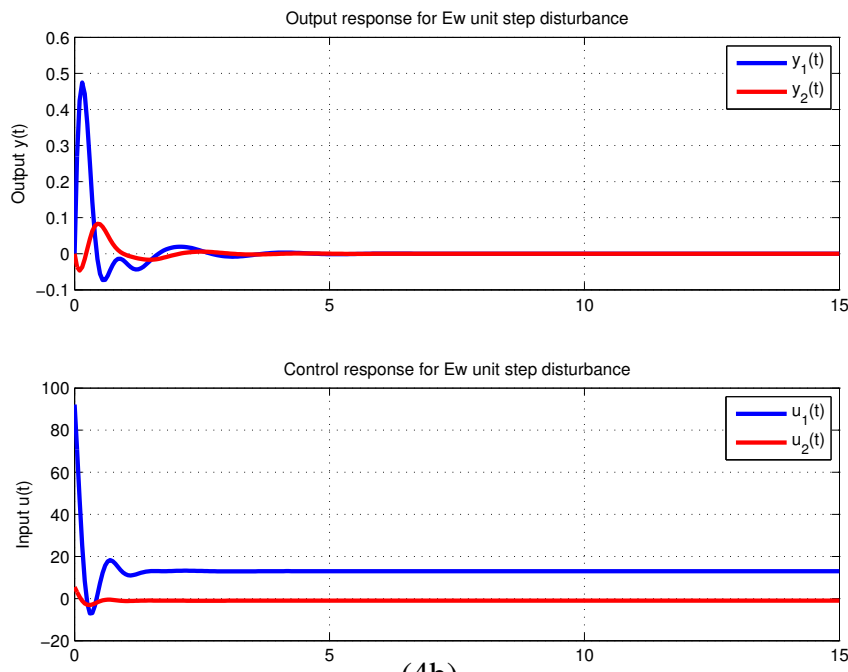

(4b)

Fig. 4. Plant output and control signal versus time (in seconds) for the example from [12] for a unit step disturbance using (a) the PID controller of [12] and (b) the proposed PID controller. 